\title{
Childhood maltreatment and coping in bipolar disorder
}

\author{
Ledo Daruy-Filho', Elisa Brietzke², Bruno Kluwe-Schiavon¹, Cristiane da Silva Fabres ${ }^{1}$, and \\ Rodrigo Grassi-Oliveira ${ }^{1}$
}

1. Pontifícia Universidade Católica do Rio Grande do Sul, Porto Alegre, RS, Brazil

2. Universidade Federal de São Paulo, São Paulo, SP, Brazil

\begin{abstract}
A personal history of childhood maltreatment has been associated with unfavorable outcomes in bipolar disorder (BD). The impact of early life stressors on the course of BD may be influenced by individual differences in coping skills. The coping construct relies on neurocognitive mechanisms that are usually influenced by childhood maltreatment. The objective of the present study was to verify the association between childhood maltreatment and coping skills in individuals with BD Type 1. Thirty female euthymic outpatients with BD Type 1 were evaluated using the Childhood Trauma Questionnaire and two additional instruments to measure their coping preferences: Ways of Coping Questionnaire (coping strategies) and Brief COPE (coping styles). Reports of physical abuse $(\mathrm{B}=.64, p \leq .01)$ and emotional abuse $(\mathrm{B}=.44, p=.01)$ were associated with the use of maladaptive strategies that focused on emotional control. Adaptive strategies and styles of coping, such as focusing on the problem, were chosen less frequently by women who had experienced emotional neglect $(\mathrm{B}=.53, p \leq .01)$ and physical abuse ( $\mathrm{B}=-.48, p \leq .01)$ in childhood. The small sample size in the present study prevented subgroup analyses. The sample did not include male BD participants. Our results indicate that early traumatic events may have a long-lasting deleterious influence on coping abilities in female BD patients. Future prospective studies may investigate whether the negative impact of childhood maltreatment over the course of BD is mediated by individual differences in coping abilities. Keywords: coping, child abuse, childhood trauma, bipolar disorder.
\end{abstract}

Received 28 August 2012; received in revised form 29 December 2012; accepted 21 January 2013. Available online 23 December 2013.

\section{Introduction}

Adverse experiences in childhood have been recognized as common events in individuals with bipolar disorder (BD), with approximately half $(49 \%)$ of these individuals reporting at least some form of abuse or neglect during childhood (Garno, Goldberg, Ramirez, \& Ritzler, 2005; Leverich et al., 2002). Furthermore,

Ledo Daruy-Filho (M.D., M.Sci.), Bruno Kluwe-Schiavon, and Cristiane da Silva Fabres, associate researcher, Developmental Cognitive Neuroscience Research Group, Graduate Program in Psychology, Human Cognition, Pontifícia Universidade Católica do Rio Grande do Sul, Porto Alegre, RS, Brazil. Elisa Brietzke ( M.D., Ph.D.), associate researcher, Program for Recognition and Intervention in Individuals with At-Risk Mental States, Department of Psychiatry, Universidade Federal de São Paulo, São Paulo, Brazil, and Interdisciplinary Laboratory of Clinical Neuroscience, Department of Psychiatry, Universidade Federal de São Paulo, São Paulo, Brazil. Rodrigo Grassi-Oliveira (M.D., $\mathrm{Ph}$.D.), professor of psychology, school of Pontifícia Universidade Católica do Rio Grande do Sul, Porto Alegre, RS, Brazil, and head of Developmental Cognitive Neuroscience Research Group, Graduate Program in Psychology, Human Cognition, Pontifícia Universidade Católica do Rio Grande do Sul, Porto Alegre, RS, Brazil. Correspondence regarding this article should be directed to: Ledo Daruy Filho, Avenida Ipiranga, 6681, prédio 11, sala 936, Partenon, Porto Alegre, RS, 90619-900, Brazil. Phone: +55 51 3320-3633 ext. 7740. E-mail: daruyfilho@gmail.com a history of childhood maltreatment (CMT) has been associated with unfavorable characteristics of BD (Alvarez, Roura, Oses, Foguet, Sola, \& Arrufat, 2011; Angst, Gamma, Rossler, Ajdacic, \& Klein, 2011; Daruy-Filho, Brietzke, Lafer, \& Grassi-Oliveira, 2011; Etain, Henry, Bellivier, Mathieu, \& Leboyer, 2008; McIntyre et al., 2008), including early age of onset (Carballo et al., 2008; Dienes, Hammen, Henry, Cohen, \& Daley, 2006), recurrence (Brown, McBride, Bauer, \& Williford, 2005), and decreased response to treatment (Marchand, Wirth, \& Simon, 2005).

Childhood maltreatment has also been significantly associated with impairment in cognitive performance among various samples. The neurocognitive domains that appear to be associated with childhood trauma are memory (Grassi-Oliveira, Ashy, \& Stein, 2008; GrassiOliveira, Stein, Lopes, Teixeira, \& Bauer, 2008; Ritchie et al., 2011), attention, and executive function (Viola, Tractenberg, Pezzi, Kristensen, \& Grassi-Oliveira, 2013). Neuroimaging findings indicate that CMT is associated with a reduction of the volume of the hippocampus, amygdala, and anterior cingulate cortex in clinical and nonclinical human samples (Bremner et al., 1997; Stein, Koverola, Hanna, Torchia, \& McClarty, 1997; Treadway, Grant, Ding, Hollon, Gore, \& Shelton, 2009; Vythilingam et al., 2002; Woon, \& Hedges, 2008). 
A robust body of evidence indicates that exposure to early life stressors, not just CMT, can negatively impact the clinical course of BD (Kapczinski et al., 2008; Post, \& Leverich, 2006). This impact is exerted through changes in immune, endocrine, and molecular mechanisms that modulate neuroplasticity (Bender, Alloy, Sylvia, Urosevic, \& Abramson, 2010; GrassiOliveira et al., 2008; Kauer-Sant'Anna et al., 2007) and may be influenced by individual differences in stress responsivity. Interestingly, one's coping abilities may be impaired by the effects of stress mediators on relevant neurofunctional circuits (Grassi-Oliveira, Daruy-Filho, \& Brietzke, 2010).

According to Folkman, Lazarus, Gruen, \& DeLongis (1986), coping comprises the set of mechanisms used by an individual to deal with a stressful situation. These mechanisms include direct strategies for problem resolution, cognitive reevaluation, acceptance, and social support rather than nonadaptive alternatives, such as avoidance, denial, or emotional thought (Compas, 2006). Coping functions include the ability to evaluate situations and make the best decision possible, and some authors have postulated that coping is intimately linked to cognitive function. Because of this, impairments in the integrity of these mechanisms could be associated with inefficient coping strategies.

Childhood abuse and neglect create neurodevelopmental toxicity that consequently impairs all of the functions that are intimately linked to cognitive function, including coping skills (Barker-Collo, Read, \& Cowie, 2012; Del-Ben, Vilela, de Crippa, Hallak, Labati, \& Zuardi, 2001; Grassi-Oliveira et al., 2008; Sesar, Simic, \& Barisic, 2010). As a result, abused and neglected individuals may likely use less adaptive forms of stress management and infrequently use coping strategies that focus on the resolution of problems. The objective of the present study was to verify the existence of an association between CMT and coping strategies in individuals with BD Type 1 .

\section{Methods}

Female outpatients with BD Type 1 and without psychiatric comorbidities, aged 18 to 65 years, were selected from an outpatient unit of the Hospital Materno-Infantil Presidente Vargas in Porto Alegre, Brazil. The diagnoses of BD and comorbidities were confirmed using the Semi-structured Clinical Interview for the Diagnostic and Statistical Manual of Mental Disorders, 4th edition (SCID-I-CV; Del-Ben et al., 2001). Only euthymic patients were included, with euthymia defined as a score on the Young Mania Rating Scale $\leq 7$ and 21-item Hamilton Depression Rating Scale $\leq 7$ (Hamilton, 1960; Vilela, Crippa, Del-Ben, \& Loureiro, 2005; Young, Biggs, Ziegler, \& Meyer, 1978). Patients with diagnoses of other mental disorders, such as psychotic disorders, substance abuse or dependence, and dementia, or other organic disorders were excluded. All of the procedures for data collection were conducted by a trained research team.
Before their inclusion in the study, all of the participants provided written informed consent. The investigation protocol was approved by the ethics committee of the Hospital Materno-Infantil Presidente Vargas and Pontifícia Universidade Católica do Rio Grande do Sul.

\section{Coping}

Coping was evaluated using the BrazilianPortuguese versions of the Ways of Coping Questionnaire (WCQ) and Brief COPE, two self-applied instruments. The WCQ (Folkman et al., 1986; Seidl, Tróccoli, \& Zannon, 2001) comprises a set of 45 items, in which the frequency of use of different coping strategies is presented in a Likert format, from 1 ("I never do this") to 5 ("I always do this"). The participant is asked to select a specific stress-related event and, using a 5-point Likerttype scale, indicate how he would respond to that event. The coping strategies are divided into four factors: $(i)$ focused on the problem (i.e., active efforts to manage, cope, solve, or reappraise the problem), (ii) focused on emotion (i.e., efforts to regulate the emotional states associated with the stressor as a way to reduce emotional discomfort without the objective to solve the problem; these include emotional reactions, such as rage, anxiety, guilt, avoidance, and passive behavior), (iii) religious or fantastic thought (i.e., religious behavior, thoughts, and faith that help when coping with problems), and (iv) search for social support (i.e., actively search for information or emotional support).

The Brief COPE (Carver, 1997; Ribeiro, \& Rodrigues, 2004) is a Likert-type, self-applied instrument that consists of a set of 28 items that cover 14 coping styles in the original version and nine coping styles in the Portuguese version. The individual is asked how he managed stressful situations, from 0 ("I never did this") through 3 ("I did this a lot"). After factorial analysis with samples of BD patients, the original 14 factors were grouped into three independent factors: $(i)$ focused on the problem (i.e., active coping, planning positive reinterpretation, acceptance, and search for instrumental, emotional, and social support), (ii) adaptive and focused on emotion (i.e., apply strategies of self-distraction, humor, expression of feelings, and religious thought), and (iii) maladaptive and focused on emotion (i.e., use of a substance to cope, such as alcohol and drugs, denial, guilt, and behavioral disengagement). This factorial regrouping was performed with the objective of adapting the results to the theoretical constructs proposed by the authors.

\section{Childhood maltreatment}

Ahistory of CMT was evaluated using the Childhood Trauma Questionnaire (CTQ; Grassi-Oliveira, Stein, \& Pezzi, 2006). The CTQ is a self-applied instrument that evaluates the impact of traumatic events that occurred in adolescents and adults during childhood. The CTQ is a set of 28 items that investigates five subscales of traumatic events: (i) physical abuse, (ii) emotional 
abuse, (iii) sexual abuse, (iv) physical neglect, and (v) emotional neglect. The questionnaire is presented in a Likert-type scale that represents how frequently events were experienced, from 1 (Never) to 5 (significant frequency or always), resulting in a score from 5 to 25 for each of the five components of the scale. In the present study, the cutoffs adopted were proposed by the cohort of Walker et al. (1999) and intended to identify a positive history of CMT (Walker et al., 1999).

\section{Statistical analyses}

A one-sample Kolmogorov-Smirnov test was used to test the normality of all of the continuous variables. To elucidate relationships between the factors of the WCQ and Brief COPE and between coping factors and CMT, Pearson's correlation test was performed. In this case, for variables that were not normally distributed, Spearman's correlation test was performed. Stepwise linear regression analysis was performed to identify independent factors (CMT) associated with the coping factors of the WCQ and Brief COPE. Values of $p<.05$ were considered statistically significant. The data were analyzed using SPSS version 16.0 (SPSS, Chicago, IL, USA).

\section{Results}

Thirty female patients with BD Type 1 were included in the study. The demographic variables, distributions of coping strategies, and histories of CMT are presented in Table 1.

Table 1. Description of the sample $(n=30)$.

\begin{tabular}{ll}
\hline & Mean (SD) \\
\hline Clinical and Demographic Data & \\
Age (years) & $43.77(12.36)$ \\
Age of first mood episode (years) & $26.07(11.23)$ \\
Duration of illness (years) & $17.6(10.65)$ \\
Number of episodes & $4.75(3.32)$ \\
Number of hospitalizations & $3.77(4.5)$ \\
HAM-D (21-item) score & $5.57(4.7)$ \\
YMRS score & $2.9(3.88)$ \\
Childhood Maltreatment & \\
Components of CTQ (5-25) & \\
Emotional abuse & $14.96(5.67)$ \\
Emotional neglect & $13.63(5.24)$ \\
Physical abuse & $10.86(5.53)$ \\
Physical neglect & $9.63(4.46)$ \\
Sexual abuse & $7.43(4.40)$ \\
Total CTQ (25-125) & $56.53(18.67)$ \\
Coping & \\
WCQ (1-5) & \\
Religious or fantastic thoughts & $3.32(.76)$ \\
Focused on the problem & $3.06(.67)$ \\
Focused on emotion & $2.98(.77)$ \\
Search for emotional support & $2.76(.84)$ \\
Brief COPE (0-3) & \\
Focused on the problem & $1.65(.59)$ \\
Adaptive and focused on emotion & $1.51(.58)$ \\
Maladaptive & $1.12(.49)$ \\
\hline
\end{tabular}

HAM-D, Hamilton Depression Rating Scale; YMRS, Young Mania Rating Scale; CTQ, Childhood Trauma Questionnaire; WCQ, Ways of Coping Questionnaire.
Emotional abuse was the most frequent type of CMT in the sample, with $80 \%$ of the individuals reporting some type of emotional abuse during childhood. More than half of the females reported a history of physical abuse and neglect $(66.7 \%$ and $63.3 \%$, respectively). Emotional neglect during childhood was found in $43.3 \%$ of the individuals, and $26.7 \%$ of those individuals reported sexual abuse.

The use of fantastic thoughts or religious practices was the most frequent modality for coping measured by the WCQ, which was used by two-thirds of the sample. A similar frequency was found using the Brief COPE. The WCQ indicated that $56 \%$ of the patients frequently used strategies that were focused on managing specific stressors, with responses including "sometimes," "frequently," and "always." Forty-six percent of the strategies were focused on emotion, and $33 \%$ were focused on the search for social support. The most frequently used coping styles (i.e., "sometimes" and "frequently") for individuals assessed using the Brief COPE were focusing on the problem $(66.7 \%)$ and focusing on emotion (46.7\%). The maladaptive style of coping was the least frequent style used by the individuals in the sample (16.7\%).

\section{Coping measurements: ways of coping questionnaire and brief COPE}

The regrouping of the 14 original factors of the Brief COPE into three factors that showed similarities to the WCQ is presented in Table 2. The two factors of the Brief COPE that suggested styles of adaptive coping were found to be associated with the WCQ factors "focused on the problem" and "religious or fantastic thought." The search for social support was inversely associated with coping strategies focused on emotion. Maladaptive coping and a focus on emotion were directly related to the WCQ's coping strategy that focused on emotion.

Table 2. Correlation between factors of the WCQ and Brief COPE.

\section{Brief COPE}

\begin{tabular}{|c|c|c|c|c|}
\hline & & $\begin{array}{l}\text { Focused } \\
\text { on the } \\
\text { problem }\end{array}$ & $\begin{array}{c}\text { Adaptive } \\
\text { and focused } \\
\text { on emotion }\end{array}$ & $\begin{array}{l}\text { Maladap- } \\
\text { tive and } \\
\text { focused on } \\
\text { emotion }\end{array}$ \\
\hline \multirow{4}{*}{$\begin{array}{l}0 \\
0 \\
3\end{array}$} & Focused on the problem & $.39^{\mathrm{b}}$ & $.49^{\mathrm{a}}$ & $-.39^{\mathrm{b}}$ \\
\hline & Focused on emotion & $-.53^{a}$ & -.07 & $.54^{\mathrm{a}}$ \\
\hline & $\begin{array}{l}\text { Fantastic or religious } \\
\text { thoughts }\end{array}$ & .13 & $.41^{\mathrm{b}}$ & -.00 \\
\hline & Search for social support & $.38^{\mathrm{b}}$ & .20 & .03 \\
\hline
\end{tabular}

\section{Coping and childhood maltreatment}

Table 3 shows the results of the associations between coping and a history of CMT in the exploratory correlational analysis. The severity of CMT was generally positively associated with a preference for 
Table 3. Exploratory analysis between coping and childhood maltreatment $(n=30)$.

\begin{tabular}{|c|c|c|c|c|c|c|}
\hline & $\begin{array}{l}\text { Total } \\
\text { CTQ* }\end{array}$ & $\begin{array}{l}\text { Physical } \\
\text { neglect * }\end{array}$ & $\begin{array}{l}\text { Emotional } \\
\text { neglect * }\end{array}$ & $\begin{array}{l}\text { Sexual } \\
\text { abuse }{ }^{* *}\end{array}$ & $\begin{array}{l}\text { Physical } \\
\text { abuse * }\end{array}$ & Emotional abuse * \\
\hline \multicolumn{7}{|l|}{ WCQ } \\
\hline Focused on the problem & -.31 & -.02 & $-.53^{\mathrm{a}}$ & $.40^{\mathrm{b}}$ & $-.45^{\mathrm{b}}$ & -.34 \\
\hline Focused on emotion & $.42^{\mathrm{b}}$ & .20 & .28 & .19 & .33 & $.44^{\mathrm{b}}$ \\
\hline Fantastic and religious thought & -.13 & -.09 & -.29 & $.43^{\mathrm{b}}$ & -.18 & -.10 \\
\hline Search for social support & -.01 & .04 & -.29 & $.38^{\mathrm{b}}$ & -.06 & -.70 \\
\hline \multicolumn{7}{|l|}{ Brief COPE } \\
\hline Focused on the problem & $-.42^{b}$ & -.25 & $-.49^{\mathrm{a}}$ & .02 & -.29 & -.34 \\
\hline Adaptive and focused on emotion & $-.52^{a}$ & $-.46^{\mathrm{a}}$ & $-.48^{\mathrm{a}}$ & .02 & $-.48^{a}$ & $-.44^{b}$ \\
\hline Maladaptive and focused on emotion & $.60^{\mathrm{a}}$ & $.36^{\mathrm{b}}$ & $.55^{\mathrm{a}}$ & .13 & $.64^{\mathrm{a}}$ & $.44^{\mathrm{b}}$ \\
\hline
\end{tabular}

WCQ, Ways of Coping Questionnaire. *Pearson Correlation, ${ }^{* *}$ Spearman Correlation, ${ }^{\mathrm{a}} p \leq .01,{ }^{\mathrm{b}} p \leq .05$.

coping styles and strategies that centered on emotional reactions rather than on more adaptive coping strategies that focused on problem-solving.

The pattern of coping as related to emotional expression was associated with a history of neglect (physical or emotional) and physical and emotional abuse. Additionally, the use of coping strategies that focused on the problem was negatively associated with the presence of physical abuse and emotional neglect.

The use of adaptive coping strategies and styles that focus on emotion, evaluated by the Brief COPE, showed an inverse relationship with the severity of CMT, especially with regard to emotional neglect, whereas the presence of childhood sexual abuse was related only to an adaptive pattern of coping measured by the WCQ.

The objective of the present study was to evaluate the impact of the severity of CMT on the preference for coping styles and strategies, for which a multiple regression analysis was conducted (Tables 4 and 5). Each factor of the WCQ and Brief Cope was included as a dependent variable in the equations, and the subscales of the CTQ were considered independent variables. The subscale for sexual abuse was excluded from the regression because its results did not present a normal distribution with strong positive asymmetry.

A linear relationship was found between the frequency of emotional neglect during childhood on the WCQ and the decreased use of coping strategies that focused on the problem (Table 4). Similarly, the severity of emotional abuse was directly proportional to the frequency of using coping strategies that focused on emotional control.

Table 5 shows a similar linear regression, but it included data from the Brief COPE. Participants who were subjected to physical abuse during childhood presented coping styles that appeared to depend on the frequency and severity of abuse. More importantly, however, is the frequent use of maladaptive coping strategies that focused on emotion rather than the less frequent use of adaptive coping strategies. The frequency of emotional neglect during childhood was found to be a significant predictor of the infrequent implementation of coping strategies that focused on a resolution of the stressor.

Table 4. Linear regression: Coping (WCQ) and history of childhood maltreatment.

\begin{tabular}{|c|c|c|c|c|c|c|}
\hline & $R$ & $\Delta R^{2}$ & $\Delta F$ & $d f$ & $B$ & $p$ \\
\hline Focused on the problem & .53 & .25 & 11.08 & 1.29 & & .002 \\
\hline Physical neglect & & & & & .33 & ns \\
\hline Emotional neglect & & & & & -.53 & .002 \\
\hline Physical abuse & & & & & -.14 & ns \\
\hline Emotional abuse & & & & & .07 & ns \\
\hline Focused on emotion & .44 & .16 & 6.78 & 1.29 & & .01 \\
\hline Physical neglect & & & & & -.00 & ns \\
\hline Emotional neglect & & & & & -.05 & ns \\
\hline Physical abuse & & & & & .10 & ns \\
\hline Emotional abuse & & & & & .44 & .01 \\
\hline Fantastic or religious thought & .33 & -.03 & .78 & 4.29 & & ns \\
\hline Search for social support & .42 & .05 & 1.40 & 4.29 & & ns \\
\hline
\end{tabular}

.ns, nonsignificant; WCQ, Ways of Coping Questionnaire.

\section{Discussion}

The present results support the hypothesis that traumatic events during childhood negatively interfere with the way adult individuals with BD cope with stress. Bipolar patients who were subjected to CMT, especially physical neglect and physical abuse, presented a preference for coping styles and strategies that focused on emotional control and were associated with the cognitive reappraisal of problems and strategies that included the avoidance of coping. Furthermore, the styles and strategies associated with problem solving were less frequently used in the subpopulation of BD patients who were subjected to CMT. 
Table 5. Linear regression: Coping (Brief COPE) and history of childhood maltreatment.

\begin{tabular}{|c|c|c|c|c|c|c|}
\hline & $R$ & $\Delta R^{2}$ & $\Delta F$ & $d f$ & $B$ & $p$ \\
\hline Focused on the problem & .49 & .22 & 9.20 & 1.29 & & .005 \\
\hline Physical neglect & & & & & .00 & ns \\
\hline Emotional neglect & & & & & -.49 & .005 \\
\hline Physical abuse & & & & & .12 & ns \\
\hline Emotional abuse & & & & & .02 & ns \\
\hline $\begin{array}{l}\text { Adaptive and focused on } \\
\text { emotion }\end{array}$ & .48 & .21 & 8.76 & 1.29 & & .006 \\
\hline Physical neglect & & & & & -.31 & ns \\
\hline Emotional neglect & & & & & -.28 & ns \\
\hline Physical abuse & & & & & -.48 & .006 \\
\hline Emotional abuse & & & & & -.23 & ns \\
\hline $\begin{array}{l}\text { Maladaptive and focused on } \\
\text { emotion }\end{array}$ & .64 & .39 & 19.6 & 1.29 & & .000 \\
\hline Physical neglect & & & & & .11 & ns \\
\hline Emotional neglect & & & & & .18 & ns \\
\hline Physical abuse & & & & & .64 & .004 \\
\hline Emotional abuse & & & & & .08 & ns \\
\hline
\end{tabular}

ns, nonsignificant

One important aspect is the difference between the instruments used to evaluate coping. The WCQ is applied based on one specific stressor chosen by the patient and is based on the model of coping strategies by Folkman et al. (1986). This approach provides evidence of the way the individual copes with a specific problem. The Brief COPE, in contrast, evaluates the most common way to cope with stressors (coping traits or coping styles; Carver, Scheier, \& Weintraub, 1989). Both concepts evaluated by WCQ and Brief COPE were similar showing association with CMT.

Reducing the 14 factors from the Brief COPE to three factors was compatible with the theoretical framework presented by the WCQ. An important difference between these two instruments is the strategies that focus on emotion. In the WCQ, the factor "coping focused on emotion" combines several strategies related to emotional control, such as anger and guilt, which imply intrinsic dysfunctional characteristics. In the Brief COPE, the authors considered that certain strategies (e.g., humor and distraction) are functional despite being focused on emotion. Because of this, a specific factor for those strategies was created.

Furthermore, the present results are consistent with previous studies that used samples from different populations. Walsh, Fortier, \& Dilillo (2010) found a relationship between a history of childhood sexual abuse and emotion-oriented coping in a sample of university students. Sesar et al. (2010) and Hager, \& Runtz (2012) studied coping and CMT and proposed that avoidance and denial strategies are among the more common coping strategies.

Our hypothesis suggests that the relationship between CMT and dysfunctional coping is based on the impact of severe stress during critical periods of neurodevelopment, thus impairing cognition and affecting memory (Ritchie et al., 2011), attention, and executive function (Savitz et al., 2008). These findings are corroborated by structural neuroimaging studies that described volumetric reductions of the hippocampus (Bremner et al., 1997; Stein et al., 1997; Woon, \& Hedges, 2008). Additionally, the size of the amygdala was found to be reduced in a pediatric population who was exposed to maltreatment (Weniger, Lange, Sachsse, \& Irle, 2008), although increased activity was also found (Grant, Cannistraci, Hollon, Gore, \& Shelton, 2011). Moreover, the mediators involved in the neuroprogression of $\mathrm{BD}$, such as inflammation and neurotrophic factors (Berk, 2011), may be influenced by CMT. In fact, CMT is associated with reprogramming of the release of brain-derived neurotrophic factor (Elzinga, 2011), and a close association between traumatic events and cytokines has been observed (Guo, 2012).

The hippocampus, amygdala, and prefrontal cortex may be vulnerable to several secondary mechanisms that may compromise their functionality, resulting in difficulties in coping strategies that depend on the integrity of certain cognitive processes, particularly processes related to the resolution of problems and cognitive reappraisal, such as executive function, all of which depend on the prefrontal cortex (Clark, Rogers, Armstrong, Rakowski, \& Kviz, 2008; Funahashi, 2001; Tanji, \& Hoshi, 2008). Additionally, the activity of the amygdala, in association with the prefrontal cortex, can modulate emotional responses to ensure that behavior is appropriate for the specific context (Bachevalier, \& Malkova, 2006; Garrett, \& Chang, 2008). Individuals with amygdalar hyperactivity associated with prefrontal dysfunction exhibited behaviors and thoughts with more emotional characteristics when dealing with stressors (i.e., coping strategies focused on emotion).

Based on the present results, we hypothesize that BP patients exposed to CMT will use less adaptive strategies in a probable stressful situation in the future, rather than strategies directed toward problem resolution. Therefore, BP patients will tend to experience the stressor in a more negative way, thereby increasing the activation of the mediators involved in the neurophysiology of stress (Bender et al., 2010; Kauer-Sant'Anna et al., 2007) and allostatic load (Kapczinski et al., 2008). These facts can postulate the moderating role of coping between CMT and bipolar disorder progression. Thus, a new focus for psychosocial interventions may be introduced that is centered on readjusting coping strategies to relieve the progressive nature of the disorder (Grassi-Oliveira, Daruy-Filho, \& Brietzke, 2010).

The limitations of the present study include the small sample size and its restriction to women. More studies are necessary to elucidate specific cognitive processes associated with coping strategies and the neuroanatomical and neurofunctional processes impacted by traumatic events during childhood that affect the strategies chosen by an individual. Moreover, 
prospective studies may investigate individual differences in coping abilities as a modulator of the impact of CMT on BD.

\section{References}

Alvarez, M. J., Roura, P., Oses, A., Foguet, Q., Sola, J., \& Arrufat, F. X. (2011). Prevalence and clinical impact of childhood trauma in patients with severe mental disorders. Journal of Nervous and Mental Disease, 199(3), 156-161.

Angst, J., Gamma, A., Rossler, W., Ajdacic, V., \& Klein, D. N. (2011). Childhood adversity and chronicity of mood disorders. European Archives of Psychiatry and Clinical Neuroscience, 261(1), 21-27.

Bachevalier, J., \& Malkova, L. (2006). The amygdala and development of social cognition: theoretical comment on Bauman, Toscano, Mason, Lavenex, and Amaral (2006). Behavioral Neuroscience, 120(4), 989-991.

Barker-Collo, S., Read, J., \& Cowie, S. (2012). Coping strategies in female survivors of childhood sexual abuse from two Canadian and two New Zealand cultural groups. Journal of Trauma \& Dissociation, 13(4), 435-447.

Bender, R. E., Alloy, L. B., Sylvia, L. G., Urosevic, S., \& Abramson, L. Y. (2010). Generation of life events in bipolar spectrum disorders: a re-examination and extension of the stress generation theory. Journal of Clinical Psychology, 66(9), 907-926.

Berk, M., Kapczinski, F., Andreazza, A. C., Dean, O. M., Giorlando, F., Maes, M., Yücel, M., Gama, C. S., Dodd, B., Magalhães, P. V. S., Amminger, P., McGorry, P., \& Malhi, G. S. (2011). Pathways underlying neuroprogression in bipolar disorder: Focus on inflammation, oxidative stress and neurothophic factors. Neurosciendce and Biobehavioral Reviews, 35(3), 804-17.

Bremner, J. D., Randall, P., Vermetten, E., Staib, L., Bronen, R. A., Mazure, C., Capelli, S., McCarthy, G., Innis, R. B., \& Charney, D. S. (1997). Magnetic resonance imaging-based measurement of hippocampal volume in posttraumatic stress disorder related to childhood physical and sexual abuse: a preliminary report. Biological Psychiatry, 41(1), 23-32.

Brown, G. R., McBride, L., Bauer, M. S., \& Williford, W. O. (2005). Impact of childhood abuse on the course of bipolar disorder: a replication study in U.S. veterans. Journal of Affective Disorders, 89(1-3), 57-67.

Carballo, J. J., Harkavy-Friedman, J., Burke, A. K., Sher, L., BacaGarcia, E., Sullivan, G. M., Grunebaum, M. F., Parsey, R. V., Mann, J. J., \& Oquendo, M. A. (2008). Family history of suicidal behavior and early traumatic experiences: additive effect on suicidality and course of bipolar illness? Journal of Affective Disorders, 109(1-2), 57-63.

Carver, C. S. (1997). You want to measure coping but your protocol's too long: consider the brief COPE. International Journal of Behavioral Medicine, 4(1), 92-100.

Carver, C. S., Scheier, M. F., \& Weintraub, J. K. (1989). Assessing coping strategies: a theoretically based approach. Journal of Personality and Socical Psychology, 56(2), 267-283.

Clark, M. A., Rogers, M. L., Armstrong, G. F., Rakowski, W., \& Kviz, F. J. (2008). Differential response effects of data collection mode in a cancer screening study of unmarried women ages $40-75$ years: a randomized trial. BMC Medical Research Methodology, 8, 10.

Compas, B. E. (2006). Psychobiological processes of stress and coping: implications for resilience in children and adolescentscomments on the papers of Romeo \& McEwen and Fisher et al. Annals of the New York Academy of Science, 1094, 226-234.

Daruy-Filho, L., Brietzke, E., Lafer, B., \& Grassi-Oliveira, R. (2011). Childhood maltreatment and clinical outcomes of bipolar disorder. Acta Psychiatrica Scandinavica, 124, 427-434.

Del-Ben, C. M., Vilela, J. A., de Crippa, J. A. S., Hallak, J. E. C., Labati, C. M., \& Zuardi, A. W. (2001). Reliability of the Structured Clinical Interview for DSM-IV - Clinical Version translated into Portuguese. Brazilian Journal of Psychiatry, 23(3), 156-159.

Dienes, K. A., Hammen, C., Henry, R. M., Cohen, A. N., \& Daley, S. E. (2006). The stress sensitization hypothesis: understanding the course of bipolar disorder. Journal of Affective Disorders, 95(1-3), 43-49.
Elzinga, B. M., Molendijk, M. L., Voshaar, R. C. O., Bus, B. A. A., Prickaerts, J., Spinhoven, P., \& Penninx, B. J. W. H. (2011). The impact of childhood abuse and recent stress on serum brain-derived neurotrophic factor and the moderating role of BDNF Val ${ }^{66} \mathrm{Met}$. Psychopharmacology, 214(1), 319-28.

Etain, B., Henry, C., Bellivier, F., Mathieu, F., \& Leboyer, M. (2008). Beyond genetics: childhood affective trauma in bipolar disorder. Bipolar Disorder, 10(8), 867-876.

Folkman, S., Lazarus, R. S., Gruen, R. J., \& DeLongis, A. (1986). Appraisal, coping, health status, and psychological symptoms. Journal of Personality and Social Psychology, 50(3), 571-579.

Funahashi, S. (2001). Neuronal mechanisms of executive control by the prefrontal cortex. Neuroscience Research, 39(2), 147-165.

Garno, J. L., Goldberg, J. F., Ramirez, P. M., \& Ritzler, B. A. (2005). Impact of childhood abuse on the clinical course of bipolar disorder. British Journal of Psychiatry, 186, 121-125.

Garrett, A., \& Chang, K. (2008). The role of the amygdala in bipolar disorder development. Development and Psychopathology, 20(4), 1285-1296.

Grant, M. M., Cannistraci, C., Hollon, S. D., Gore, J., \& Shelton, R. (2011). Childhood trauma history differentiates amygdala response to sad faces within MDD. Journal of Psychiatric Research, 45(7), 886-895.

Grassi-Oliveira, R., Ashy, M., \& Stein, L. M. (2008). Psychobiology of childhood maltreatment: effects of allostatic load? Revista Brasileira de Psiquiatria, 30(1), 60-68.

Grassi-Oliveira, R., Daruy-Filho, L., \& Brietzke, E. (2010). New perspectives on coping in bipolar disorder. Psychology \& Neuroscience, 3(2), 161-165.

Grassi-Oliveira, R., Stein, L. M., Lopes, R. P., Teixeira, A. L., \& Bauer, M. E. (2008). Low plasma brain-derived neurotrophic factor and childhood physical neglect are associated with verbal memory impairment in major depression: a preliminary report. Biological Psychiatry, 64(4), 281-285.

Grassi-Oliveira, R., Stein, L. M., \& Pezzi, J. C. (2006). [Translation and content validation of the Childhood Trauma Questionnaire into Portuguese language]. Revista de Sa úde Pública, 40(2), 249-255.

Guo, M., Liu, T., Guo, J. C., Jiang, X. L., Chen, F., \& Gao, Y. S. (2012). Study on serum cytokine levels in posttraumatic stress disorder patients. Asian Pacific Journal of Tropical Medicine, 5(4), 323-5.

Hager, A. D., \& Runtz, M. G. (2012). Physical and psychological maltreatment in childhood and later health problems in women: an exploratory investigation of the roles of perceived stress and coping strategies. Child Abuse \& Neglect, 36(5), 393-403.

Hamilton, M. (1960). A rating scale for depression. Journal of Neurology, Neurosurgery and Psychiatry, 23, 56-62.

Kapczinski, F., Vieta, E., Andreazza, A. C., Frey, B. N., Gomes, F. A., Tramontina, J., Kauer-Sant'anna, M., Grassi-Oliveira, R., \& Post, R. M. (2008). Allostatic load in bipolar disorder: implications for pathophysiology and treatment. Neuroscience and Biobehavioral Reviews, 32(4), 675-692.

Kauer-Sant'Anna, M., Tramontina, J., Andreazza, A. C., Cereser, K., da Costa, S., Santin, A., Yathan, L. N. \& Kapczinski, F. (2007). Traumatic life events in bipolar disorder: impact on BDNF levels and psychopathology. Bipolar Disorder, 9(Suppl. 1), 128-135.

Leverich, G. S., McElroy, S. L., Suppes, T., Keck, P. E., Jr., Denicoff, K. D., Nolen, W. A., Altshuler, L. L., Rush, A. J., Kupka, R., Frye, M. A., Autio, K. A., \&. Post, R. M. (2002). Early physical and sexual abuse associated with an adverse course of bipolar illness. Biological Psychiatry, 51(4), 288-297.

Marchand, W. R., Wirth, L., \& Simon, C. (2005). Adverse life events and pediatric bipolar disorder in a community mental health setting. Community Mental Health Journal, 41(1), 67-75.

McIntyre, R. S., Soczynska, J. K., Mancini, D., Lam, C., Woldeyohannes, H. O., Moon, S., Konarski, J. Z., \& Kennedy, S. H. (2008). The relationship between childhood abuse and suicidality in adult bipolar disorder. Violence and Victims, 23(3), 361-372.

Post, R. M., \& Leverich, G. S. (2006). The role of psychosocial stress in the onset and progression of bipolar disorder and its comorbidities: the need for earlier and alternative modes of therapeutic intervention. Development and Psychopathology, 18(4), 1181-1211. 
Ribeiro, J., \& Rodrigues, A. (2004). Questões acerca do coping: a propósito do estudo de adaptação do BRIEF Cope. Psicologia, Saúde e Doenças, 5(1), 3-15.

Ritchie, K., Jaussent, I., Stewart, R., Dupuy, A. M., Courtet, P., Malafosse, A., \& Ancelin, M. L. (2011). Adverse childhood environment and late-life cognitive functioning. International Journal of Geriatric Psychiatry, 26, 503-510.

Savitz, J. B., van der Merwe, L., Stein, D. J., Solms, M., \& Ramesar, R. S. (2008). Neuropsychological task performance in bipolar spectrum illness: genetics, alcohol abuse, medication and childhood trauma. Bipolar Disorder, 10(4), 479-494.

Seidl, E. M. F., Tróccoli, B. T., \& Zannon, C. M. L. C. (2001). Análise fatorial de uma medida de estratégias de enfrentamento. Psicologia: Teoria e Pesquisa, 17(3), 225-234.

Sesar, K., Simic, N., \& Barisic, M. (2010). Multi-type childhood abuse, strategies of coping, and psychological adaptations in young adults. Croatian Medical Journal, 51(5), 406-416.

Stein, M. B., Koverola, C., Hanna, C., Torchia, M. G., \& McClarty, B. (1997). Hippocampal volume in women victimized by childhood sexual abuse. Psychological Medicine, 27(4), 951-959.

Tanji, J., \& Hoshi, E. (2008). Role of the lateral prefrontal cortex in executive behavioral control. Physiological Review, 88(1), 37-57.

Treadway, M. T., Grant, M. M., Ding, Z., Hollon, S. D., Gore, J. C., \& Shelton, R. C. (2009). Early adverse events, HPA activity and rostral anterior cingulate volume in MDD. PLoS One, 4(3), e4887.

Vilela, J. A., Crippa, J. A., Del-Ben, C. M., \& Loureiro, S. R. (2005). Reliability and validity of a Portuguese version of the Young Mania Rating Scale. Brazilian Journal of Medical and Biological Research, 38(9), 1429-1439.
Viola, T. W., Tractenberg, S. G., Pezzi, J. C., Kristensen, C. H., \& Grassi-Oliveira, R. (2013) Childhood physical neglect associated with executive functions impairments in crack cocaine-dependent women. Drug Alcohol Depend, 132(1-2), 271-276. doi: 10.1016/j. drugalcdep.2013.02.014

Vythilingam, M., Heim, C., Newport, J., Miller, A. H., Anderson, E., Bronen, R., Brummer, M., Staib, L., Vermetten, E., Charney, D. S., Nemeroff, C. B., \& Bremner, J. D. (2002). Childhood trauma associated with smaller hippocampal volume in women with major depression. American Journal of Psychiatry, 159(12), 2072-2080.

Walker, E. A., Gelfand, A., Katon, W. J., Koss, M. P., Von Korff, M., Bernstein, D., \& Russo, J. (1999). Adult health status of women with histories of childhood abuse and neglect. American Journal of Medicine, 107(4), 332-339.

Walsh, K., Fortier, M. A., \& Dilillo, D. (2010). Adult coping with childhood sexual abuse: a theoretical and empirical review. Aggression and Violent Behavior, 15(1), 1-13.

Weniger, G., Lange, C., Sachsse, U., \& Irle, E. (2008). Amygdala and hippocampal volumes and cognition in adult survivors of childhood abuse with dissociative disorders. Acta Psychiatrica Scandinavica, $118(4), 281-290$

Woon, F. L., \& Hedges, D. W. (2008). Hippocampal and amygdala volumes in children and adults with childhood maltreatment-related posttraumatic stress disorder: a meta-analysis. Hippocampus, 18(8), 729-736.

Young, R. C., Biggs, J. T., Ziegler, V. E., \& Meyer, D. A. (1978). A rating scale for mania: reliability, validity and sensitivity. British Journal of Psychiatry, 133, 429-435. 